\title{
Measurements of the Transverse Wakefields Due to Varying Collimator Characteristics.*
}

\author{
S. Molloy ${ }^{\dagger}$, Sergei Seletskiy, Mike Woods, SLAC, Menlo Park, California \\ Jonathan David Andrew Smith, Cockcroft Institute, Warrington, UK \\ Carl David Beard, Juan Luis Fernandez-Hernando, STFC/DL/ASTeC, Daresbury, Warrington, UK \\ Nigel Watson, STFC/RAL/ASTeC, Chilton, Didcot, UK \\ Adriana Bungau, UMAN, Manchester, UK \\ Andre Sopczak, University of Lancaster, UK
}

\begin{abstract}
We report on measurements of the transverse wakefields induced by collimators of differing characteristics. An apparatus allowing the insertion of different collimator jaws into the path of a beam was installed in End Station A (ESA) in SLAC. Eight comparable collimator geometries were designed, including one that would allow easy comparison with previous results, and were installed in this apparatus. Measurements of the beam kick due to the collimator wakefields were made with a beam energy of 28.5 $\mathrm{GeV}$, and beam dimensions of 100 microns vertically and a range of 0.5 to $1.5 \mathrm{~mm}$ longitudinally. The trajectory of the beam upstream and downstream of the collimator test apparatus was determined from the outputs of ten BPMs (four upstream and six downstream), thus allowing a measurement of the angular kick imparted to the beam by the collimator under test. The transverse wakefield was inferred from the measured kick. The different aperture designs, data collection and analysis, and initial comparison to theoretical and analytic predictions are presented here.
\end{abstract}

\section{INTRODUCTION}

It is known that the collimation system at the ILC may introduce short-range wakes, which have the potential to dilute the emittance, and amplify incoming position jitter. In order to design collimators to have minimal impact on the beam quality it is necessary to fully understand how the characteristics of the collimator jaws influence the impedance seen by the beam.

Previous studies [1] have shown the complexity of analytical calculations of the impedance of particular collimator designs, even in simplified cases. Previous experimental results have advanced the ability of modeling codes such as MAFIA to predict measured data to within a factor of 2-3, however, it is important for ILC design that this agreement be improved to the $10 \%$ level.

This experimental program aims to measure the beam kick induced by various collimator designs, and to compare

\footnotetext{
* Work supported in part by the EC under the FP6 Research Infrasctructure Action - Structuring the European Research Area EUROTeV DS Project Contract no.011899 RIDS and by U.S. Department of Energy contract DEAC02- 76SF00515.

† smolloy@slac.stanford.edu
}

with theoretical predictions, and simulations of the interaction. Collimators with a range of different geometries, surface finishes, and materials were installed on the beamline in End Station A (ESA) [2] in SLAC, and their effect on the beam was measured. Results from these measurements will be compared with $3 \mathrm{D}$ computer simulations.

\section{MEASUREMENT TECHNIQUE}

The collimators were installed on the ESA beamline, and up- and down-stream beam position monitors (BPMs) were used to determine the incoming and outgoing trajectory of the beam in order to allow a measurement of the kick angle imparted by the transverse wakes. The beamline apparatus containing the collimators is detailed in [3], and a schematic is shown in figure 1 .

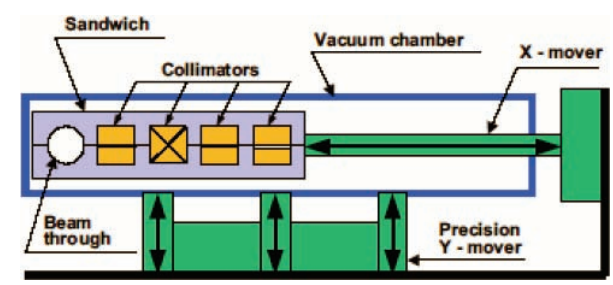

Figure 1: Schematic of the collimator wakefield measurement box (beam moving out of the page).

Figure 1 shows an inner 'sandwich' containing five possible slots for the beam to move through. Four of the slots are occupied with collimators, and the fifth is empty to allow for undisrupted beam operation. The sandwich is pushed back and forth by the X-mover in order to change the slot presented to the beam. This assembly is contained within a vacuum chamber, which is controlled by three movers. These are installed in such a way as to allow high precision movement in $y, z$, and $d y / d z$ (where $y$ is vertical, and $z$ is in the direction of motion of the beam). Further details of the mover scheme can found in [4]. Since these motors allow control and readback of the vertical position of the collimator jaws, it is unnecessary to control the beam, therefore, the beam could be centred between the collimator jaws, and the box moved to different positions to measure the change in the kick imparted to the beam.

For each set of collimator jaws, the beam was centred using upstream position feedbacks. A 'reference' dataset 
(typically 300 machine pulses) was recorded without moving the collimators in order to account for offsets of the BPMs. The collimator box was then stepped from $-1 \mathrm{~mm}$ to $+1 \mathrm{~mm}$ in $200 \mu \mathrm{m}$ steps, recording $30 \mathrm{~s}$ of data at each step. Since ESA operates at $10 \mathrm{~Hz}$, this resulted in 300 measurements at each collimator position in the case of no machine trips.

For each pulse, the readings from each of the BPMs (four upstream and six downstream) were recorded, together with the bunch's charge and energy (the energy was measured using a BPM in a high dispersion region of the machine).

\section{DATA ANALYSIS}

The kick given to each pulse was determined by performing a straight line fit to the BPMs upstream of the collimator, and a separate straight line fit to those downstream. For each dataset, the resolution of each BPM was measured by comparing its readback to the position predicted from the output of the other BPMs. The calculated resolution was used as the weighting factor in the two straight line fits. The kick imparted to the beam was calculated as the difference in the slopes of these fits.

Some pulses with zero or very low charge, indicating a 'misfire' of the beam, were removed. Also removed were pulses with unusual large BPM values, implying a BPM malfunction, or a machine error. In some early datasets it was necessary to cut on the chi-squared value of the upand down-stream fits, however, this proved unnecessary in later runs due to improved BPM performance.

\section{COLLIMATORS}

The collimators tested are shown in figures 2, 3, 4, and 5 .

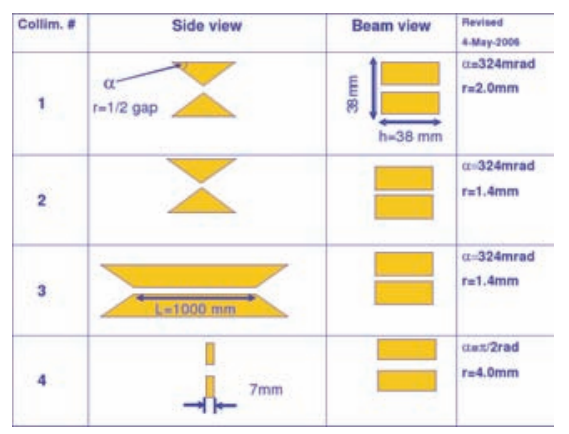

Figure 2: Schematics of the collimators from sandwich 1.

\section{EXPERIMENTAL RESULTS}

The results obtained for slots 2 and 3 have been plotted in figures 6 and 7. Since the kick should be an odd function with respect to the position of the beam (with an offset term to take into account any initial offset of the beam), the data have been fit to a third order polynomial, where the

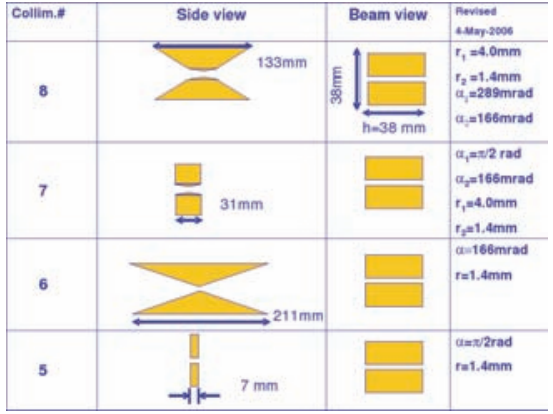

Figure 3: Schematics of the collimators from sandwich 2.

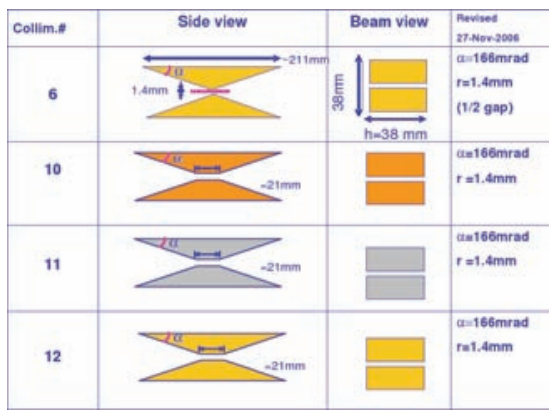

Figure 4: Schematics of the collimators from sandwich 3.

quadratic dependence has been set to zero. Since the kick is expected to be linear in the central region of the collimator gap, some of the data were fit to a straight line (after exclusion of the outer points). Full results, along with a comparison with theory and 3D modeling code, are shown in table 1 .

Table 1 show two calculations of the measured kick factor: one with a $3^{\text {rd }}$ order fit as described above, and a second with a linear fit performed on the central points. This preliminary comparion shows some agreement between simulations and measurements and further studies are ongoing. The measured result for collimator \#1 agrees well with the measurement in [1] of a collimator with the same specifications.

The results of the linear fit to the measurements have substantially lower error bars than the $3^{\text {rd }}$ order fit. This may be a consequence of the kick near the centre of the col-

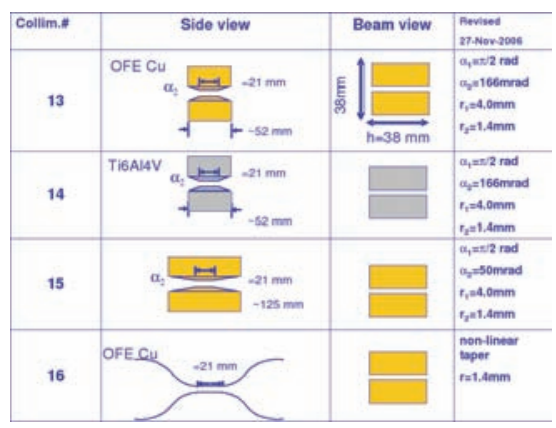

Figure 5: Schematics of the collimators from sandwich 4. 
Table 1: Measured kick factors ( $\chi^{2} / D O F$ of fit in brackets) and theoretical predictions (assuming a $500 \mu \mathrm{m}$ bunch length) Collimators $10,11, \& 12$ have not been included as their analysis is not yet complete. The 3D predictions for collimators 13-16 do not include the resistive component of the kick.

\begin{tabular}{|c|c|c|c|c|}
\hline Coll. & $\begin{array}{c}\text { Measured Kick } \\
\text { Factor / V/pC/mm } \\
\text { (Linear Fit) }\end{array}$ & $\begin{array}{c}\text { Measured Kick } \\
\text { Factor / V/pC/mm } \\
\text { (Linear \& Cubic Fit) }\end{array}$ & $\begin{array}{c}\text { Analytic Prediction } \\
\text { Kick Factor } \\
\text { V/pC/mm }\end{array}$ & $\begin{array}{c}\text { 3-D Modeling } \\
\text { Prediction Kick } \\
\text { Factor V/pC/mm }\end{array}$ \\
\hline \hline 1 & $1.4 \pm 0.1(1.0)$ & $1.2 \pm 0.3(1.0)$ & 2.27 & $1.63 \pm 0.37$ \\
\hline 2 & $1.4 \pm 0.1(1.3)$ & $1.2 \pm 0.3(1.4)$ & 4.63 & $2.88 \pm 0.84$ \\
\hline 3 & $4.4 \pm 0.1(1.5)$ & $3.7 \pm 0.3(0.8)$ & 5.25 & $5.81 \pm 0.94$ \\
\hline 4 & $0.9 \pm 0.2(0.8)$ & $0.5 \pm 0.4(0.8)$ & 0.56 & 0.8 \\
\hline 5 & $3.7 \pm 0.1(7.9)$ & $4.9 \pm 0.2(2.6)$ & 4.59 & 6.8 \\
\hline 6 & $0.9 \pm 0.1(0.9)$ & $0.9 \pm 0.3(1.0)$ & 4.65 & $2.12 \pm 1.14$ \\
\hline 7 & $1.7 \pm 0.1(0.7)$ & $2.2 \pm 0.3(0.5)$ & 4.59 & $2.87 \pm 0.53$ \\
\hline 8 & $1.7 \pm 0.3(2.0)$ & $1.7 \pm 0.3(2.2)$ & 4.59 & $2.39 \pm 0.89$ \\
\hline 13 & & $4.1 \pm 0.4(0.8)$ & & $3.57 \pm 0.98$ \\
\hline 14 & & $2.6 \pm 0.4(1.0)$ & & $3.57 \pm 0.98$ \\
\hline 15 & & $2.0 \pm 0.3(1.8)$ & & $2.51 \pm 1.16$ \\
\hline 16 & & $1.3 \pm 0.3(1.0)$ & & $2.35 \pm 1.50$ \\
\hline
\end{tabular}

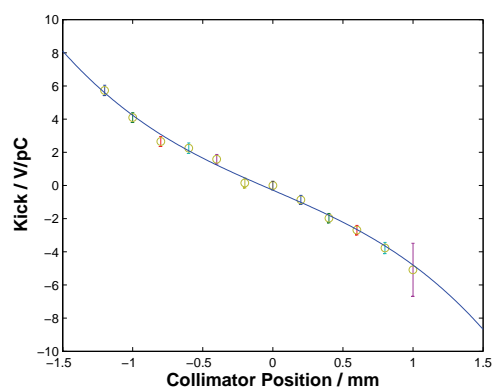

Figure 6: Kick factor vs collimator position for coll. 3.

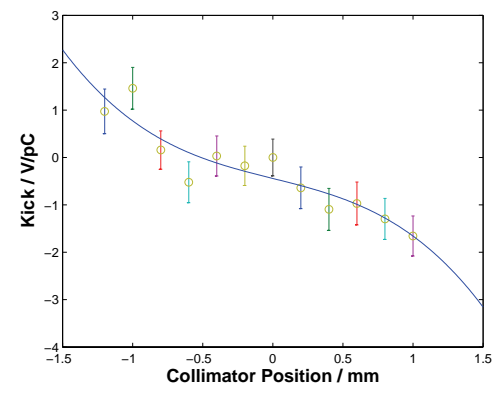

Figure 7: Kick factor vs collimator position for coll. 4.

limator having a well understood linear relationship with the beam position, while the magnitude of the kick in the region close to the collimator surface having a more complex behaviour that is poorly modeled by the cubic coefficient of a polynomial fit.

Also noticeable are substantial differences between the measured results, the analytical predictions, and the 3D simulations. Many of the 3D simulations agree with the measurements within their associated errors, however there are still some anomalies in the data. For example, it is important that it is understood why the measurements of collimators \#1 and \#2 agree, despite these collimators being the same specifications but with different half-gaps. Also, it is not yet understood why collimator \#14 has a lower measured kick factor than \#13, despite being of the same shape, but constructed from a material of higher resistivity (note that the 3D simulations are identical for these collimators as the resistive component was not simulated).

\section{CONCLUSIONS}

A robust collimator wakefield measurement system has been developed at SLAC's ESA, and this has been used to measure a total of 15 collimators. Analytical calculations and $3 \mathrm{D}$ computer simulations have been performed to predict the kick factors induced by these collimators, although some of this work is currently ongoing.

Disparities between the measurements and the predictions have been found, and work is underway to determine their source.

\section{REFERENCES}

[1] P. Tenenbaum et al, "Direct measurement of the transverse wakefields of tapered collimators", PRST-AB, Jan 9, 2007.

[2] M. Woods et al, "A Test Facility for the International Linear Collider at SLAC End Station A for Prototypes of Beam Delivery and IR Components", PAC'05, Knoxville, TN.

[3] P. Tenenbaum et al, "An apparatus for the direct measurement of collimator transverse wakefields", PAC'99, New York, NY.

[4] G. Bowden et al, "Precision magnet movers for the Final Focus Test Beam”, SLAC-PUB-95-6132

[5] P. Tenenbaum et al., contribution TUP3A03, Proc. EPAC-00 (2000). 